\title{
Perceptions variétales et usages médicinaux de Irvingia gabonensis par les populations autochtones de deux départements au sud du Bénin
}

\author{
S.D. Osseni, F.P. Tchobo, A.J. Djossou, M.M. Soumanou \\ Sawab Deen OSSENI, Unité de Recherche en Génie Enzymatique et Alimentaire, Laboratoire d'Étude et de Recherche \\ en Chimie Appliquée. École Polytechnique d'Abomey-Calavi, Université d'Abomey-Calavi, Bénin, 01 BP 2009 Tél : (+229) \\ 960041 48/63 4799 59, E-mail : sawabdeenosseni@yahoo.fr, calavi, République du Bénin. \\ Dr. (MC) Fidèle Paul TCHOBO, Unité de Recherche en Génie Enzymatique et Alimentaire, Laboratoire d'Étude et de \\ Recherche en Chimie Appliquée. École Polytechnique d'Abomey-Calavi, Université d'Abomey-Calavi, Bénin, 01 BP 2009 \\ Tél : (+229) 976862 01, E-mail : fideletchobo@gmail.com, calavi, République du Bénin \\ Dr. Andriano Jospin DJOSSOU, Unité de Recherche en Génie Enzymatique et Alimentaire, Laboratoire d'Étude et de \\ Recherche en Chimie Appliquée. École Polytechnique d'Abomey-Calavi, Université d'Abomey-Calavi, Bénin, 01 BP 2009 \\ Tél : (+229) 961250 83, E-mail : jospindjossou@gmail.com, calavi, République du Bénin \\ Professeur Mohamed Mansourou SOUMANOU, Unité de Recherche en Génie Enzymatique et Alimentaire, Laboratoire \\ d'Étude et de Recherche en Chimie Appliquée. École Polytechnique d'Abomey-Calavi, Université d'Abomey-Calavi, \\ Bénin, 01 BP 2009 Tél : (+229) 97877870, E-mail : msoumanoufr@yahoo.fr, calavi, République du Bénin
}

Original submitted in on $7^{\text {th }}$ June 2018. Published online at www.m.elewa.org on $30^{\text {th }}$ September 2018 http://dx.doi.org/10.4314/jab.v129i1.14

\section{RÉSUMÉ}

Objectif : Irvingia gabonensis (pomme sauvage) est une espèce présente au Bénin tout comme dans d'autres régions de l'Afrique de l'Ouest et du Centre.. La présente étude porte sur les perceptions variétales, l'importance socio-économique et culturelle de Irvingia gabonensis dans le Couffo et le Plateau, deux départements au sud du Bénin. L'objectif est de montrer les différentes perceptions variétales ainsi que les différents usages médicinaux que les populations autochtones de ces départements font de cette espèce Méthodologie et résultats : L'étude a été réalisée sur un échantillon de 131 personnes possédant des arbres de Irvingia gabonensis. Le logiciel sphinx 2 a servi à analyser les données. Les enquêtes ont révélé d'une part qu'il existait deux variétés du fruit de Irvingia gabonensis et d'autre part que la commercialisation de ses fruits générait d'importantes devises pour les producteurs dans ces régions. Outre l'intérêt économique que génère le fruit, il est à noter que pour l'obtention de l'amande, plus de $64 \%$ des enquêtés utilisent la pulpe comme engrais organique ce qui accroitrait la productivité des cultures

Conclusion et application des résultats: II existe dans les deux départements, deux variétés Irvingia gabonensis. Les organes de cette plante sont aussi utilisés en pharmacopée traditionnelle. lls sont utilisés dans la cicatrisation des plaies, dans le traitement des maux de ventre, du paludisme, de l'anémie, des œdèmes et des plaies intestinales. La décoction aqueuse est la forme pharmaceutique la plus citée.

Mots clés : Irvingia gabonensis, perception variétale, médecine traditionnelle, Bénin 
Varietal perceptions and medicinal uses of Irvingia gabonensis by indigenous populations in two departments in southern Benin

\section{ABSTRACT}

Objective : Irvingia gabonensis (bush mango) is a species present in Benin as well as in other parts of West and Central Africa. The present study discusses to varietal perceptions, the socio-economic and socio-cultural importance of Irvingia gabonensis in two departments of southern Benin.

Methodology and results: The study was conducted on 131 tree holders of Irvingia gabonensis in four municipalities to each of the two departments prospected. The investigations revealed, that there are two varieties of Irvingia gabonensis fruits and there marketing generates important devises to holders. In addition to the economic interest generated by the fruit, it should be noted that in order to obtain the kernel, more than $64 \%$ of the respondents use the pulp as an organic fertilizer which would increase the productivity of the crops. Conclusion and application of results: Two variety exist in this two departments. The organs of this plant are also used in traditional medicine. They are used in wound healing of the uterus after delivery, in the treatment of stomachaches, malaria, anemia, edema and intestinal wounds. The aqueous decoction is the most cited pharmaceutical form.

Keywords: Irvingia gabonensis, varietal perception, traditional medicine, Benin

\section{INTRODUCTION}

À l'instar des autres produits de l'agriculture qui assurent la sécurité alimentaire des populations et qui contribuent au développement économique des pays de l'Afrique, les produits forestiers non ligneux alimentaires occupent aussi une place de choix. $\mathrm{Ce}$ sont des plantes sauvages ou cultivées dont le potentiel utilitaire a été peu exploité commercialement, mais qui constituent un support de survie économique et alimentaire pour les populations locales (Gandari, 2008). D'après l'Organisation Mondiale de la Santé, les plantes « sauvages " participent à la satisfaction des besoins en matière de santé et d'alimentation pour $80 \%$ des personnes qui vivent dans les pays en développement (Andel, 2006). À l'échelle planétaire, la FAO (2004 a) précise que 1,6 milliard de personnes dépendent des ressources forestières pour vivre et que 1,2 milliard d'entre elles vivant dans les pays en développement, utilisent ou exploitent les arbres pour l'alimentation ou la commercialisation. Leurs intérêts biochimiques et nutritionnels (beaucoup sont riches en protéines, lipides, glucides, minéraux et de diverses vitamines et fibres) (Augustino et al., 2011) ont démontré leur potentiel à résoudre les crises alimentaires, nutritionnelles et sanitaires croissants en Afrique (Okolo et al., 1995 ; Chadare et al., 2010 ; Ahenkan et Boon., 2011 ; Sossa et Vihotogbé et al., 2012). Au
Bénin, une importance particulière est accordée à Irvingia gabonensis depuis des lustres particulièrement à l'amande. Le genre Irvingia est rencontré au Bénin (Sokpon et Lejoly, 1996) et produit aussi bien des qualités de fruits à mésocarpe allant du goût très sucré à l'amer avec même un aspect gluant (Vihotogbé, 2001). Le caractère comestible ou non de la pulpe de Irvingia gabonensis donne lieu à deux variétés: Irvingia gabonensis var. gabonensis (caractérisée par un mésocarpe sucré et comestible), et Irvingia gabonensis var. excelsa (à mésocarpe amer et non comestible) (Harris, 1996 ; Tchoundjeu et al, 2005). Irvingia gabonensis pousse le mieux sur des sols acides bien drainés dans les forêts denses et humides où les précipitations annuelles atteignent $1500-3000 \mathrm{~mm}$; dans ces conditions, Irvingia gabonensis peut atteindre $40 \mathrm{~m}$ de haut (kengni et al., 2011). Irvingia gabonensis est présent au sud du Bénin (où elle est plus abondante), notamment dans les départements du Mono, du Couffo et dans les forêts denses de Pobè (Akouêhou, 2012) ou dans les champs en jachères et jardins de case où elle provient d'une régénération naturelle assistée ou de plantation après des germinations de semences par des agriculteurs. On estime que le marché des graines de Irvingia gabonensis représente environ 50 millions de USD de chiffre d'affaires et qu'il existe 
des échanges importants des fruits et des amandes au Nigeria, au Bénin, au Gabon et au Cameroun. Les différents usages de Irvingia gabonensis ont été abordés par plusieurs travaux. Ainsi, Okolo et al. (1995) rapportent que la décoction de l'écorce de cet arbre est efficace contre les maux de ventre et la dysenterie. Les mêmes auteurs affirment aussi que des études scientifiques ont montré l'existence de principes actifs, conférant des propriétés analgésiques à l'écorce de $I$. gabonensis, ce qui justifie son utilisation en Sierra Léone pour soulager les douleurs. De même, Ndoye et al. (1994) mentionnent que la décoction de l'écorce est aussi efficace dans le traitement de la diarrhée et dans le sevrage des enfants (Ainge et Brown, 2004). Ayuk et al., (1999) soulignent par ailleurs qu'au Cameroun la décoction de l'écorce de Irvingia gabonensis est utilisée dans le traitement de l'hernie et comme antidote pour l'empoisonnement tandis que les agriculteurs utilisent les feuilles comme fourrage. Quant aux amandes, Ngondi et al, (2005) rapportent que la consommation de l'huile qui y est extraite aurait un effet sur la régulation pondérale. Ces mêmes auteurs ont montré que des extraits de l'amande de Irvingia. gabonensis permettent de réguler la pression systolique et que le fruit constitue

\section{MATERIEL ET METHODES}

Échantillonnage des enquêtés : La réalisation de la présente étude a été précédée d'une étude prospective dans les départements du Couffo et du Plateau qui constituent deux départements de forte production de Irvingia gabonensis au sud du Bénin. Ainsi elle a permis d'évaluer les perceptions variétales, les aptitudes technologiques et les usages médicinaux auprès des populations autochtones dans quatre communes de chacun des deux départements ciblés. II s'agit des communes de Toviklin, de Lalo, d'Aplahoué, et de Klouékanmè pour le compte du département du Couffo ; de Pobè, de Kétou, d'Adja-Ouèrè, et d'Ifangni pour le compte du département du Plateau. La taille de l'échantillon des enquêtés est déterminée suivant la formule de (Dagnelie et al., 1998; Chadare et coll., 2008) :

$$
\mathrm{Ni}=4 \mathrm{Pi}(1-\mathrm{Pi}) / \mathrm{d}^{2}
$$

$\mathrm{Ni}$ : Nombre total d'individus à enquêter dans la localité i. $\mathrm{Pi}$ : Taux d'individus (connaissantl'arbre) obtenu pendant l'étude prospective dans le département i. un puissant laxatif pour les populations. De plus (Ainge et Brown, 2004) ont montré que la consommation du « Dikanut », l'amande de Irvingia gabonensis régularise le taux de glucose sanguin, et par conséquent, est efficace contre le diabète. L'arbre est aussi utilisé comme bois d'œuvre ou bois de chauffe (Ayuk et al., 1999 ; Leakey et al., 1999). Eu égard aux valeurs nutritionnelles, médicinales qu'offrent l'écorce et la feuille de Irvingia gabonensis, ainsi qu'aux aptitudes technologiques de son amande et de sa pulpe, toute recherche visant à accroître sa valeur et sa disponibilité constitue une source d'opportunités pour les ressources agricoles et forestières afin de permettre la survie des communautés rurales et urbaines. De plus, outre le caractère sucré que présente le mésocarpe de la variété gabonensis de Irvingia gabonensis, il existe au niveau des populations rurales d'autres critères d'appréciations pour distinguer ses différents fruits. Ainsi la présente étude trouve son intérêt de par son objectif qui est de montrer la perception variétale et les différentes utilisations faites de Irvingia gabonensis par les populations autochtones de deux départements au sud du Bénin.

\section{$d$ : Marge d'erreur fixée à 0,05 .}

En se basant sur la formule ci-dessus, 80 enquêtés sont sélectionnés dans le couffo et 60 dans le plateau soit un total de 140 enquêtés dans les deux départements. Ainsi le nombre d'enquêtés de chaque commune est obtenu en se référant sur l'effectif de la population de cette commune par la formule ci-après :

$T j=N i j \times X j / X$ où :

$T j$ : nombre à enquêté dans la commune $j$

Nij: Nombre total d'enquêtés dans le département i contenant la commune $j$

$X_{j}$ : effectif de la commune $j$

$X$ : population totale des communes parcourues dans le département $\mathrm{i}$

Collecte des données et analyse : Les données sur les différentes perceptions variétales, les aptitudes technologiques et les usages médicinaux de Irvingia gabonensis dans le département du Couffo et du Plateau ont été collectées principalement auprès des populations et les détenteurs des pieds de Irvingia gabonensis. Le 
questionnaire est axé essentiellement sur la connaissance des différents groupes socio-culturels, les perceptions variétales, l'importance socio-économique de l'amande, la gestion de la pulpe enlevée pour l'obtention

\section{RESULTATS ET DISCUSSION}

Caractéristiques sociodémographiques des producteurs de Irvingia gabonensis : Les principales caractéristiques sociodémographiques des producteurs enregistrées lors de cette enquête sont l'âge, le genre, le groupe ethnique, le niveau de scolarisation, les activités principales, et la taille des ménages. Dans les différentes zones d'étude retenues, les producteurs appartiennent à deux groupes ethniques. II s'agit de l'ethnie Adja (56,2\%) dans le Couffo et de l'ethnie Nago $(43,1 \%)$ dans le Plateau. La grande partie de ceux qui produisent Irvingia gabonensis est constituée de 78,5\% d'hommes dont l'activité principale est l'agriculture $(86,2 \%)$. La tranche d'âge des enquêtés est située entre 20 et plus de 60 ans. Par ailleurs, quelques femmes (21,50\%) ont des pieds de Irvingia gabonensis dans leurs champs. Essentiellement analphabète, la majorité $(82,3 \%)$ des enquêtés affirme n'avoir jamais été à l'école. Ces résultats sont similaires au taux départemental d'analphabétisme des adultes qui est de $85,3 \%$ rapporté par le PNUD (2008) dans son étude sur le développement humain au Bénin.

Connaissance générale des producteurs sur les différentes variétés de Irvingia gabonensis et de l'amande et l'utilisation des différentes parties de Irvingia gabonensis à des fins médicinales. Les fiches d'enquêtes ont été dépouillées, encodées et les données analysées par le logiciel Sphinx plus 2.

utilisation de sa pulpe : L'espèce Irvingia gabonensis est une espèce connue par les différentes populations enquêtées dans les départements du Couffo et du Plateau. Communément appelé « Ato » en langue adja, elle est appelée « Oro » en langue nago. Deux variétés ont été identifiées par les populations de ces départements et celles-ci les différencient essentiellement par leur teneur en eau et leur teneur en fibre. Ainsi l'une des deux variétés est appelée « Eshito » en langue adja et «Oro Olomi » en langue nago car elle contient à leur entendement beaucoup d'eau et l'autre "Ewoto» en langue adja et «Oro Onichou » en nago car elle contient moins d'eau. D'un département à un autre, les informations relatives à la production des deux variétés (nombre de pieds par commune), à la variété dominante et à la gestion de la pulpe varient. Le tableau 1 présente le nombre moyen de pieds de Irvingia gabonensis par commune, la variété dominante dans chaque commune et les différents modes de gestion de la pulpe de ce fruit.

Tableau 1 : Profil de répartition du nombre de pieds, de la variété dominante et du mode de gestion de la pulpe d'Irvingia gabonensis en fonction des départements

\begin{tabular}{|c|c|c|c|c|c|c|c|}
\hline \multirow{2}{*}{ Départements } & \multirow{2}{*}{ Communes } & \multirow{2}{*}{$\begin{array}{l}\text { NP d'Irvingia } \\
\text { gabonensis }\end{array}$} & \multicolumn{2}{|c|}{ Variété dominante } & \multicolumn{3}{|c|}{ Gestion de la pulpe } \\
\hline & & & Eshito & Ewoto & $\begin{array}{l}\mathrm{CA} \\
(\%)\end{array}$ & $\begin{array}{l}\mathrm{CH} \\
(\%)\end{array}$ & $\begin{array}{l}\mathrm{F} \\
(\%)\end{array}$ \\
\hline Couffo & $\begin{array}{l}\text { Klouekanmè } \\
\text { Toviklin } \\
\text { Lalo } \\
\text { Aplahoué }\end{array}$ & $\begin{array}{l}448 \\
895 \\
184 \\
547\end{array}$ & $\begin{array}{c}243 \\
307 \\
97 \\
105\end{array}$ & $\begin{array}{c}205 \\
588 \\
87 \\
442\end{array}$ & $\begin{array}{c}16 \\
10 \\
0 \\
0\end{array}$ & $\begin{array}{l}35 \\
40 \\
50 \\
60\end{array}$ & $\begin{array}{l}70 \\
60 \\
60 \\
90\end{array}$ \\
\hline Plateau & $\begin{array}{l}\text { Pobè } \\
\text { Kétou } \\
\text { Adja-ouèrè } \\
\text { Ifangni }\end{array}$ & $\begin{array}{l}41 \\
16 \\
32 \\
30\end{array}$ & $\begin{array}{l}29 \\
12 \\
27 \\
18\end{array}$ & $\begin{array}{c}12 \\
4 \\
5 \\
12\end{array}$ & $\begin{array}{l}20 \\
45 \\
20 \\
10\end{array}$ & $\begin{array}{c}80 \\
70 \\
85 \\
100\end{array}$ & $\begin{array}{c}0 \\
0 \\
0 \\
15\end{array}$ \\
\hline
\end{tabular}

NP : Nombre de pieds ; CA : Consommation animale ; $\mathbf{C H}$ : Consommation humaine ; F : Fertilisant

II ressort de l'analyse de ce tableau que la production de Irvingia gabonensis dans le département du Couffo est élevée (2074 pieds de l'arbre) avec la commune de Toviklin au premier rang comparativement au département du Plateau (119 pieds de l'arbre). Ce résultat est similaire à celui obtenu par Azokpota et al., (2014). Cette forte production de Irvingia gabonensis dans ce département pourrait être due à une considération particulière que les populations de la région auraient pour cet arbre. Dans le département du Couffo, 
la variété « Ewoto » est la plus dominante avec un score de 1.322 pieds contre 752 pour la variété «Eshito». Contrairement au département du Couffo, c'est la variété « Eshito» qui domine avec un score 86 pieds contre 33 pieds pour la variété "Ewoto » dans le Plateau. Pour l'obtention de l'amande, plusieurs moyens sont utilisés. Certains affirment qu'ils laissent pourrir le fruit dans leur champ ensuite ils récupèrent les amandes après concassage de la noix. Quant à d'autres, ils brisent directement le fruit entier pour en faire sortir l'amande. Ainsi, une partie des enquêtés affirme qu'elle concasse et utilise la pulpe uniquement comme fertilisant dans leurs champs. Certains utilisent cette amande dans l'alimentation humaine ou animale.
Importance socio-économique de Irvingia gabonensis dans les départements du Couffo et du Plateau : rvingia gabonensis, à travers le fruit entier, l'amande fraiche, ainsi que l'amande séchée joue un rôle très important dans l'économie des populations des différentes communes parcourues. Le tableau 2 présente les différentes formes sous lesquelles les populations vendent les différentes parties de Irvingia gabonensis, la proportion de ceux qui l'achètent, la variation du prix au kilogramme ainsi que la quantité annuellement vendue. Les fruits entiers, les amandes fraîches et les amandes séchées sont les différentes formes de ventes des fruits de Irvingia gabonensis. Ainsi dans le département du Couffo, $46,59 \%$ des producteurs vendent le fruit en entier tandis que 51,85\% vendent l'amande séchée du fruit.

Tableau 2 : Évaluation sommaire de la commercialisation d'Irvingia gabonensis en fonction des communes

\begin{tabular}{|c|c|c|c|c|c|c|c|c|}
\hline \multirow[t]{3}{*}{ Communes } & \multicolumn{3}{|c|}{ Différentes formes de vente } & \multicolumn{2}{|c|}{$\begin{array}{c}\text { Catégories } \\
\text { d'acheteurs }\end{array}$} & \multirow{3}{*}{$\begin{array}{l}\text { Quantité } \\
\text { d'amandes } \\
\text { sèches } \\
\text { vendue/an }\end{array}$} & \multirow{2}{*}{\multicolumn{2}{|c|}{$\begin{array}{c}\text { Prix } \\
\text { unitaire/mesure } \\
\text { d'amande sèche } \\
\text { (FCFA) }\end{array}$}} \\
\hline & \multirow[t]{2}{*}{$\begin{array}{l}\mathrm{FE} \\
(\%)\end{array}$} & \multirow[t]{2}{*}{$\begin{array}{l}\text { AFF } \\
(\%)\end{array}$} & \multirow[t]{2}{*}{$\begin{array}{l}\text { ASF } \\
(\%)\end{array}$} & \multirow[t]{2}{*}{$\begin{array}{l}\text { Com } \\
(\%)\end{array}$} & \multirow[t]{2}{*}{$\begin{array}{l}\text { Cons } \\
(\%)\end{array}$} & & & \\
\hline & & & & & & & Maxi & Mini \\
\hline Klouekanmè & 50 & 0 & 50 & 70,37 & 29,63 & 1045 & 4500 & 1000 \\
\hline Toviklin & 45,71 & 0 & 54,28 & 100 & 0 & 2490 & 5000 & 800 \\
\hline Lalo & 40,63 & 6,25 & 53,13 & 78,26 & 21,74 & 582 & 4500 & 1000 \\
\hline Aplahoué & 50 & 0 & 50 & 84,21 & 15,79 & 1332 & 4000 & 1200 \\
\hline Pobè & 51,12 & 0 & 48,28 & 0 & 100 & 168 & 3500 & 800 \\
\hline Kétou & 94,5 & 0 & 5,5 & 0 & 100 & 47 & 3000 & 700 \\
\hline Adja-ouèrè & 65 & 20 & 15 & 20 & 80 & 59 & 4000 & 1000 \\
\hline Ifangni & 90 & 0 & 10 & 20 & 80 & 41 & 3500 & 1000 \\
\hline
\end{tabular}

FE : Fruit entier ; AFF : Amande fraiche du fruit ; ASF : Amande séchée du fruit ; Com : Commerçants ; Cons : Consommateurs.

Dans ce département la grande partie de la production ( $83,21 \%$ des 5.449 kilogrammes d'amandes séchées) est achetée par les commerçants, le reste $(16,79 \%)$ est acheté par les consommateurs et le prix du kilogramme varie entre 800 francs en période d'abondance et 5000 francs CFA en période de rareté du fruit. Par contre dans le département du Plateau $75,5 \%$ des producteurs vendent le fruit en entier contre $15,5 \%$ qui vendent l'amande séchée. Les grands acheteurs sont composés essentiellement des consommateurs de la zone $(90 \%)$ et des commerçants ; c'est-à-dire les revendeurs (10\%). Environ $315 \mathrm{~kg}$ d'amandes sont vendus annuellement à un prix variant entre 700 et 4000 francs CFA en fonction de la disponibilité. En effet, l'amande de Irvingia gabonensis génère des devises aux producteurs. Selon Kengni et al. (2011), les fruits sont vendus localement tandis que les amandes s'échangent au plan régional principalement entre pays de l'Afrique de l'Ouest.
Importance des différents organes de Irvingia gabonensis dans la médecine traditionnelle : Dans les deux départements enquêtés, nous pouvons noter qu'à l'issue des entretiens auprès des 131 producteurs que la feuille de Irgengia gabonensis est l'organe le plus utilisé ( $72 \%)$, suivi de l'écorce $(24,8 \%)$, et de la racine $(3,2 \%)$. Le tableau 3 présente les différents symptômes ou affections, les modes d'utilisations et les organes régulièrement utilisés en fonction des communes.En pharmacopée traditionnelle, plusieurs utilisations sont faites des différents organes de Irvingia gabonensis pour le traitement de certains maux dans les différentes communes enquêtées. II s'agit du traitement des plaies de l'utérus de la nourrice après l'accouchement, du paludisme, des anémies, des œdèmes, des plaies intestinales et des maux de ventre. Dans le département du Couffo, toutes communes confondues, la prise par voie orale de la décoction aqueuse des feuilles de Irvingia 
gabonensis accélère la cicatrisation des plaies de l'utérus de la nourrisse après accouchement selon $82,50 \%$ des enquêtés; la feuille pourrait donc avoir un pouvoir cicatrisant élevé vis-à-vis d'autres feuilles, savoir que détiendraient ces populations du sud bénin, raison pour laquelle ils l'utilisent à ces fins. $12,5 \%$ de ces mêmes enquêtés utilisent cette feuille pour traiter le paludisme et $5 \%$ s'en servent pour traiter l'anémie.

Tableau 3 : Différentes utilisations des organes de Irvingia gabonensis en fonction des communes

\begin{tabular}{|c|c|c|c|c|c|c|c|c|c|c|}
\hline \multirow[b]{2}{*}{ Traitements } & \multirow[b]{2}{*}{ Organes (\%) } & \multirow[b]{2}{*}{$\begin{array}{l}\text { Modes } \\
\text { D'utilisation }\end{array}$} & \multicolumn{4}{|c|}{ Couffo } & \multicolumn{4}{|c|}{ Plateau } \\
\hline & & & 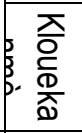 & 穴 & $\frac{\nwarrow}{\bar{\partial}}$ & $\frac{\frac{7}{\frac{7}{0}}}{\frac{0}{0}}$ & 뭄. & 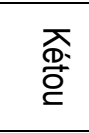 & ") & 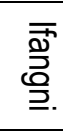 \\
\hline $\begin{array}{l}\text { Cicatrisation des plaies de } \\
\text { l'utérus de la nourrisse } \\
\text { dues à l'accouchement }\end{array}$ & $\begin{array}{l}\mathbf{F}(73,33) \\
\text { E. }(3,2)\end{array}$ & $\begin{array}{l}\text { Décoction } \\
\text { aqueuse }\end{array}$ & + & + & + & + & - & - & - & + \\
\hline Paludisme & $\begin{array}{l}\mathbf{F}(14,44) ; \\
\text { E. }(3,22)\end{array}$ & $\begin{array}{l}\text { Décoction } \\
\text { aqueuse }\end{array}$ & + & + & + & + & - & - & - & - \\
\hline Anémies & $\begin{array}{l}F(5,5) ; \\
E .(25,80)\end{array}$ & $\begin{array}{l}\text { Décoction } \\
\text { aqueuse }\end{array}$ & + & + & + & + & - & - & - & - \\
\hline OEdèmes & $\mathbf{F}(1,1)$ & $\begin{array}{l}\text { Décoction } \\
\text { aqueuse }\end{array}$ & + & + & + & + & & & & \\
\hline Plaies intestinales & $\mathbf{R}(1)$ & $\begin{array}{l}\text { Décoction } \\
\text { aqueuse + } \\
\text { Décoction de la } \\
\text { racine de èche }\end{array}$ & + & + & - & - & - & - & - & - \\
\hline Maux de ventre & E. $(67,74)$ & $\begin{array}{l}\text { Décoction } \\
\text { alcoolique }\end{array}$ & - & - & - & - & + & + & + & + \\
\hline
\end{tabular}

$\mathbf{F}$ : feuilles de Irvingia gabonensis ; E : écorce de Irvingia gabonensis ; R : racine de Irvingia gabonensis ; + : utlisée ; - : non utilisée

Dans ce département, $8,75 \%$ utilisent l'écorce pour traiter l'anémie. Par ailleurs, dans le département du Plateau, c'est plutôt pour traiter les maux de ventre que l'écorce est fortement utilisée par les populations (64\%), mais une partie des enquêtés (20\%) l'utilise aussi pour traiter l'anémie. Les différentes utilisations d'Irvingia

\section{CONCLUSION}

La présente étude a essentiellement porté sur les différentes connaissances des producteurs de Irvingia gabonensis dans le département du Plateau et du Couffo. Le département du Couffo est le département où la production de Irvingia gabonensis est élevée. Sur un échantillon de 131 enquêtés, il en ressort que les populations accordent une grande importance à la commercialisation du fruit entier, à l'amande séchée et à l'utilisation des différents organes de l'arbre à des fins médicinales. La répartition des pourcentages de réponse gabonensis en pharmacopée sont justifiées par les observations faites dans d'autres pays de la sous-région (Ngondi et al., 2005; Ainge et al., 2004 ; Ayuk et al., 1999 ; Okolo et al., 1995 ; Ndoye et al., 1994).

des enquêtés montre que les feuilles sont les plus utilisées suivies de l'écorce et de la racine. Six affections et symptômes sont traités essentiellement dans les deux groupes ethniques rencontrés. II serait donc important d'encourager la production de Irvingia gabonensis afin de valoriser au mieux la pulpe du fruit et d'initier des travaux de recherches scientifiques à travers une caractérisation physico-chimique des différents organes de cet arbre afin de permettre sa valorisation dans la médecine au Bénin.

\section{RÉFÉRENCE BIBLIOGRAPHIQUE}


Ahenkan A and Boon E, 2011. Improving Nutrition and Health through Non-timber Forest Products in Ghana. Journal of Health Population and Nutrition 29 (2): 141-148

Ainge $L$ and Brown NBM, 2004. (Irvingia gabonensis and I. wombolu) In Clark, Sunderland, TCH. 2004. The Key Non-Timber Forest Products of Central Africa:State of the Knowledge. Technical Paper No. 122, May 2004, SD Publication Series; Office of Sustainable Development; Bureau for Africa; USAID, Washington, D.C. 20523

Akouêhou GS, 2012. Évaluation et analyse socioéconomique des marchés des produits forestiers non ligneux, cas du Néré, du Karité, du Rônier, du Baobab, du Pommier sauvage et de l'Arbre à Pain: les chaines de commercialisation, les différents maillons, les caractéristiques, les différents acteurs directs et indirects, les interrelations et les différents flux dans les zones d'intervention du projet. Rapport FAO. Cotonou. $118 p$.

Andel TV, 2006. Les produits forestiers autres que le bois d'œuvre: la valeur des plantes sauvages. Wageningen, Pays-Bas : Agromisa et CTA.

Agustino S, Mataya B, Senelwa K, Achigan-Dako EG (2011) Non-Wood Forest Products and Services for Socio-Economic Development. A Compendium for Technical and Professional Forestry Education. The African Forest Forum, Nairobi, Kenya.

Ayuk ET, Duguma B, Franzel S, Kengue J, Mollet M, Tiki Manga T, Zenkeng P, 1999. Uses, management and economic potential of Irvingia gabonensis in the humid lowlands of Cameroon. Forest Ecology and Management 113 (1): 1-9

Azokpota P and Akouehou S, 2014. Valorisation du Pommier Sauvage (Irvingia Gabonensis FTE Ixon) dans les Communes de Klouékanmè, Toviklin, Lalo et Aplahoué au Bénin. Rapport FAO. Projet d'Appui à la promotion et à la valorisation des Produits Forestiers Non LigneuX» (PA-PFNL) au Bénin : (PCT/BEN/3303, Bénin, 15p.)

Chadare FJ, Hounhouigan, JD, Linnemann AR, Nout MJR, VanBoeke MAJSI, 2008. Indigenous knowledge and processing of Adansonia digitata L. food products in Benin', Ecology of Food and Nutrition vol. 47 no. 4, pp. 338-62.

Chadare FJ, Gayet DP, Azokpota P, Nout MJR, Linnemann AR, Hounhouigan JD, van Boekel
MAJS, 2010. Three Traditional Fermented Baobab Foods from Benin, Mutchayan, Dikouanyouri, and Tayohounta: Preparation, Properties, and Consumption. Ecology of Food and Nutrition 49 (4):279-297.

Dagnelie $P, 1998$. Statistiques théoriques et appliquées : Inférence statistique à une et à deux dimensions, de Boeck Université, Brussels : De Boeck and Larcier S.A., Tome 2, $559 \mathrm{p}$.

FAO, 2004 a: The state of food insecurity in the world. Monitoring progress towards the world food summit and millennium development goals. Roma: FAO

Gandari J, 2008. Les fruits indigènes. Spore, 136, 8-10

Harris DJ, 1996. A revision of the Irvingiaceae in Africa. Bulletin du Jardin Botanique National de Belgique 65 : 143-196.

Kengni E, Kengue J, Ebenezer EBK, Tabuna H, 2011. Irvingia gabonensis, Irvingia wombolu, pommier sauvage. Conservation et utilisation durable des ressources génétiques des espèces ligneuses alimentaires prioritaires de l'Afrique subsaharienne. Bioversity International (Rome, Italie).

Leakey RB, 1999. The evolution of agroforestry systems, In: Cultivating Trees, Martin GJ, Agama AL and Leakey RRB (Eds.), People and Plants Handbook 5:1-2.

Ndoye $\mathrm{O}$ and Tchamou N, 1994. Utilization and marketing trends for Irvingia gabonensis products in Cameroon. ICRAF-IITA conferences on Irvingia gabonensis, Ibadan, Nigeria. May.

Ngondi JL, Oben E, Minka SR, 2005. The effect of Irvingia gabonensis seeds on body weight and blood lipids of obese subjects in Cameroon. Lipid in Health and Disease $4: 12-15$

Okolo CO, Johnson PB, Abdurahman EM, Abdu Aguye I, Hussaini IM, 1995. Analgesic effect of $l$. gabonensis stern bark extract. Journal of Ethnopharmacology 45 (2): 125-129.

Sokpon $\mathrm{N}$ and Lejoly J, 1996. Les plantes alimentaires d'une forêt dense caducifoliée : Pobè au sud-est du Bénin. In : L'alimentation en forêt tropicale : Interactions bioculturelles et perspectives de développement (Eds Hladik CM, Hladik A, Linares OF, Oagezy H, Semple A, Hadley M) : 315-324. Man and the Biosphere Series No. 13. UNESCO, Paris, France

Sossa-Vihotogbé CNA, Akissoe NH, Anihouvi VB, Ahohuendo BC, Ahanchede A, Sanni A, 
Hounhouigan, DJ, 2012. Endogenous knowledge of four leafy vegetables used by rural populations in Benin. Ecology of Food and Nutrition 51 (1): 22-39

Tchoundjeu Z, Atangana AR, Degrande A, 2005 . Indigenous methods in preserving bush mango kernel in Cameroon. American Journal of Applied Sciences 2 (9): 1337-1342

Vihotogbe R, 2001. Diversité biologique et potentialités socio-économiques des Ressources Alimentaires Végétales-RAVs (Produits Forestiers Non Ligneux-PFNL) de la forêt de Pobè et de ces zones connexes. Mémoire d'ingénieur Agronome, Faculté des Sciences Agronomiques, Université d'Abomey-Calavi, Bénin. 